\title{
CLINICAL-EPIDEMIOLOGICAL NEUROPSYCHIATRIC STUDY OF CHORNOBYL LIQUIDATORS
}

\section{Konstantin Loganovsky, Vladimir Buzunov}

State Institution «National Research Center for Radiation Medicine of National Academy of Medical Sciences of Ukraine» 53 llyenko [Melnykov] Street, Kyiv, 04050, Ukraine, loganovsky@windowslive.com

Background: Experts of UN Chernobyl Forum recognized the long-term mental consequences as the major social and medical burden of the catastrophe. There are data on increased morbidity and mortality from cardiovascular and cerebrovascular pathology in the dose-effect manner. Goal: to conduct the risk-analysis of the neuropsychiatric pathology in clean-up workers (liquidators) of the Chornobyl catastrophe (April 26, 1986).

Subjects and Methods: Liquidators from the State Register of Ukraine with radiation doses records $(n=68,145)$ and Clinical-Epidemiological Register of the $\operatorname{NRCRM}(n=3,548)$. Clinical verification of neuropsychiatric diseases, epidemiological methods, risk-analysis.

Results: Excess of the incidence of acute cerebrovascular and organic mental disorders was found: organic psychosis (ICD-9: 293.0-294.9), (RR: 3.15, 95\% Cl 2.6-3.7); non-psychotic organic brain damage (ICD-9: 310.0-310.9; ICD-10: F07.0-F07.9), (RR: 1.99, 95\% Cl 1.6-2.5); acute cerebrovascular disorders (ICD-9: 430.0-436.9; ICD-10: I60.0-I66.0), (RR: 1.40, 95\% Cl 1.3-1.5), consequences of cerebrovascular disorders (ICD-9: 438.0-439.9; ICD-10:I69.0169.8), (RR: $1.23,95 \% \mathrm{Cl} 1.0-1.5)$. Significant radiation risks of morbidity and mortality due to acute and chronic cererbrovascular disorders in liquidators exposed at the age $<40$ years and at the dose range $>0.25$ Gy $(0.25-0.7$ Gy) were revealed.

Incidence of neuropsychiatric disorders in cleanup workers 1986-1987 depending on age at the time of exposure

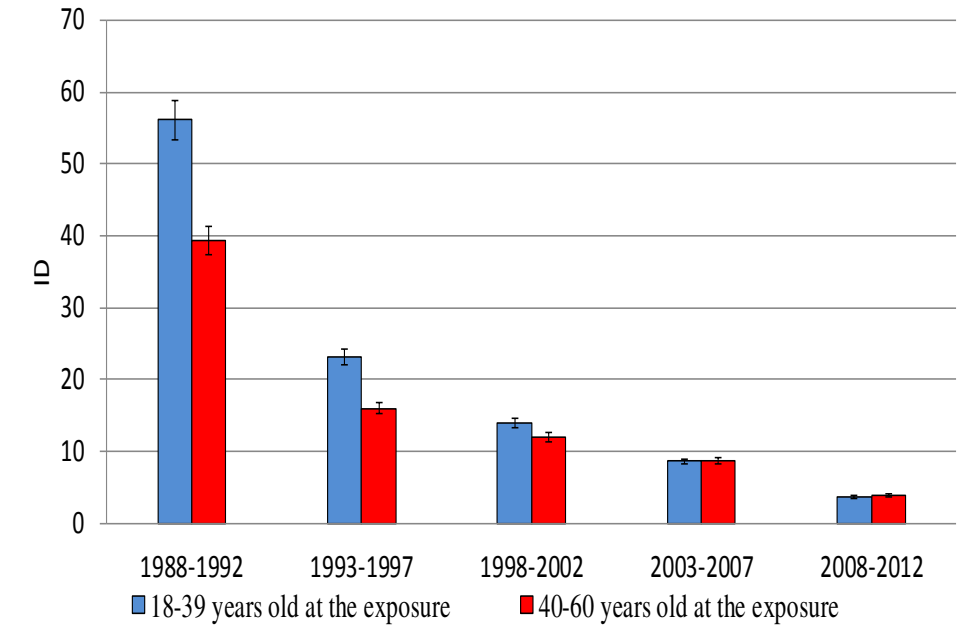

Autonomous nervous system disorders (Dysautonomia) (ICD-10:G90.0-G90.9)

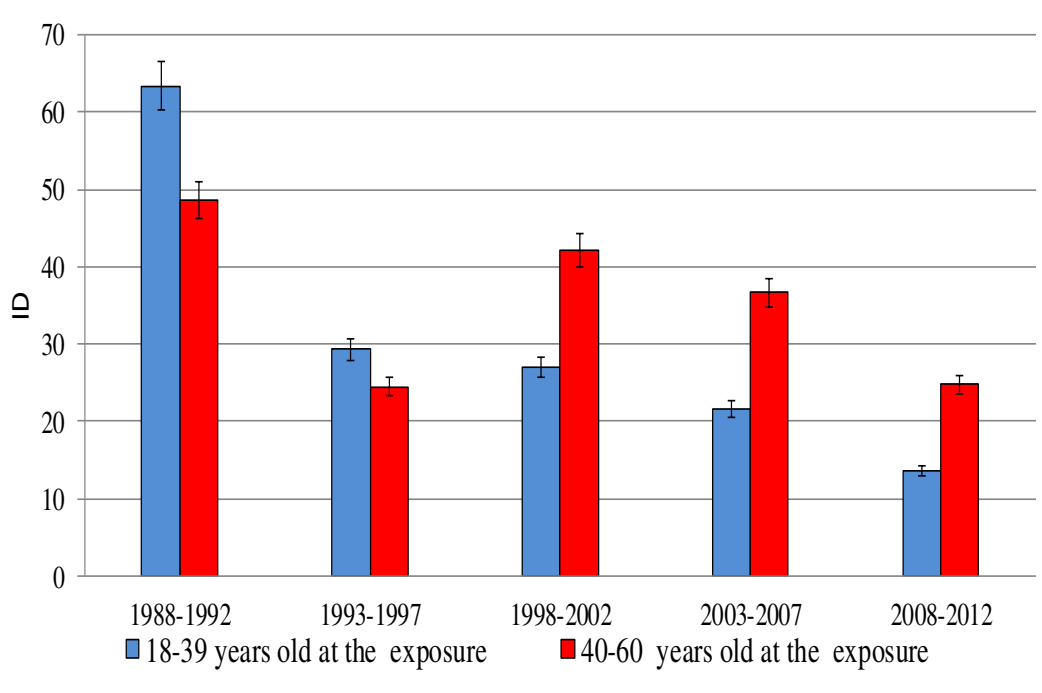

Nervous system diseases (ICD-9: 320.0-359.9, ICD-10: G00-G99)
Disease

$\mathrm{RR}(95 \% \mathrm{Cl})$

Organic psychosis

(ICD-9: 293.0-294.9)

$3.15(2.6 ; 3.7)$

Non-psychotic organic brain damage

(ICD-9: 310.0-310.9; ICD-10

F07.0-F07.9)

Acute cerebrovascular

disorders

(ICD-9: 430.0-436.9; ICD-10:

$1.40(1.3 ; 1.5)$

I60.0-166.0)

Consequences of

cerebrovascular disorders

(ICD-9: 438.0-439.9; ICD-

10:169.0-169.8)

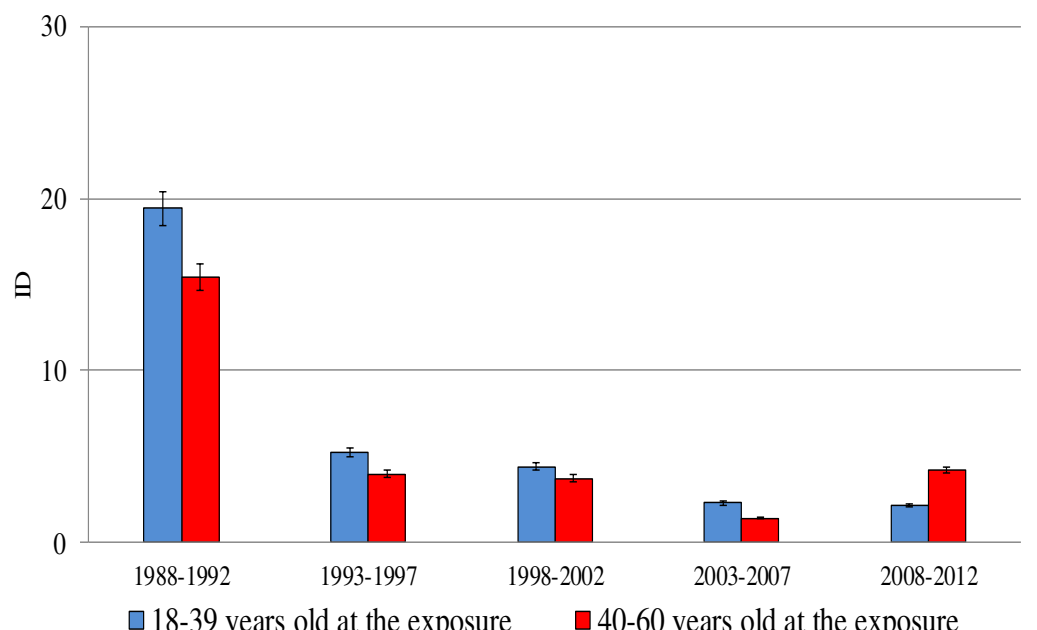

Mental and behavioral disorders (ICD-9: 290-319, ICD-10: F00-F99)

Relative Risks (RR) and 95\% Confidential Intervals (Cl) in cleanup workers 1986-1987 depending on the external radiation dose and age at the time of exposure. Period - 1988-2021, control-exposed <0,05 Gy

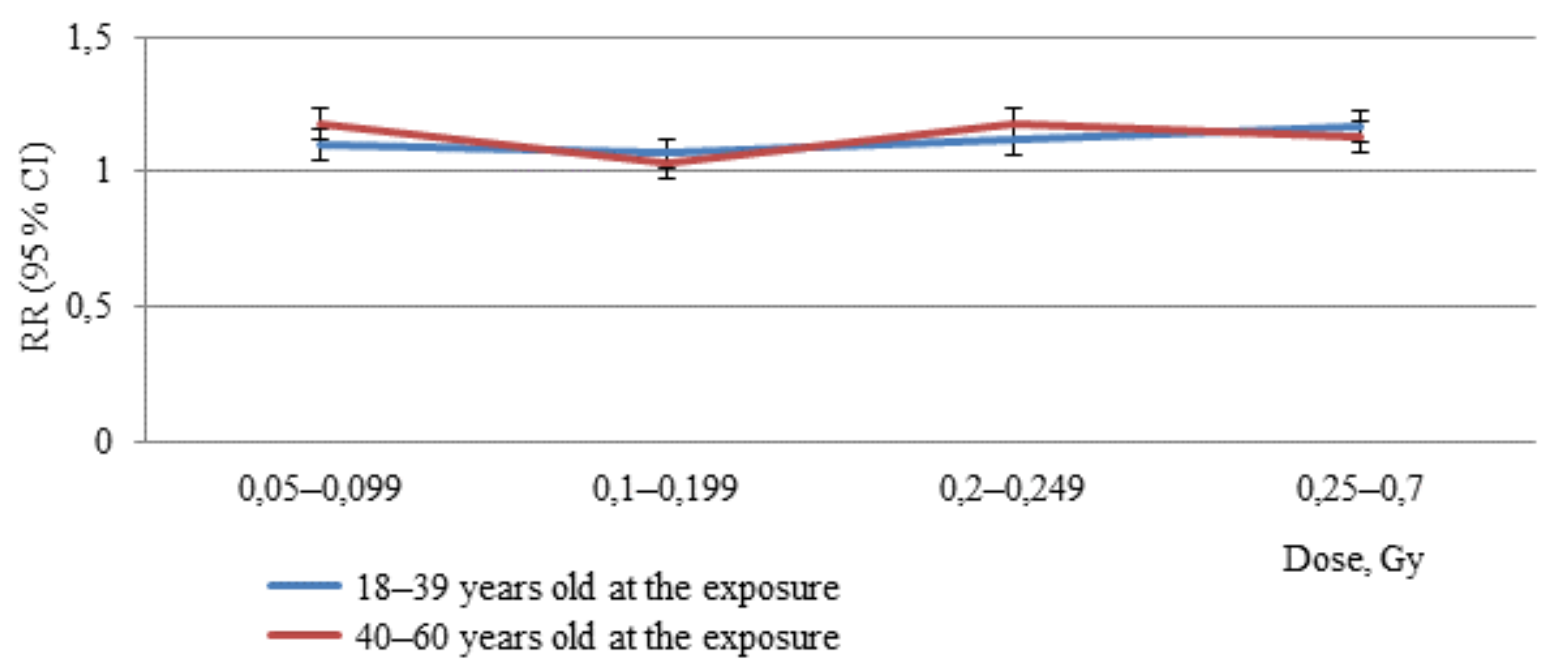

Autonomous nervous system disorders (Dysautonomia) (ICD-10:G90.0-G90.9)

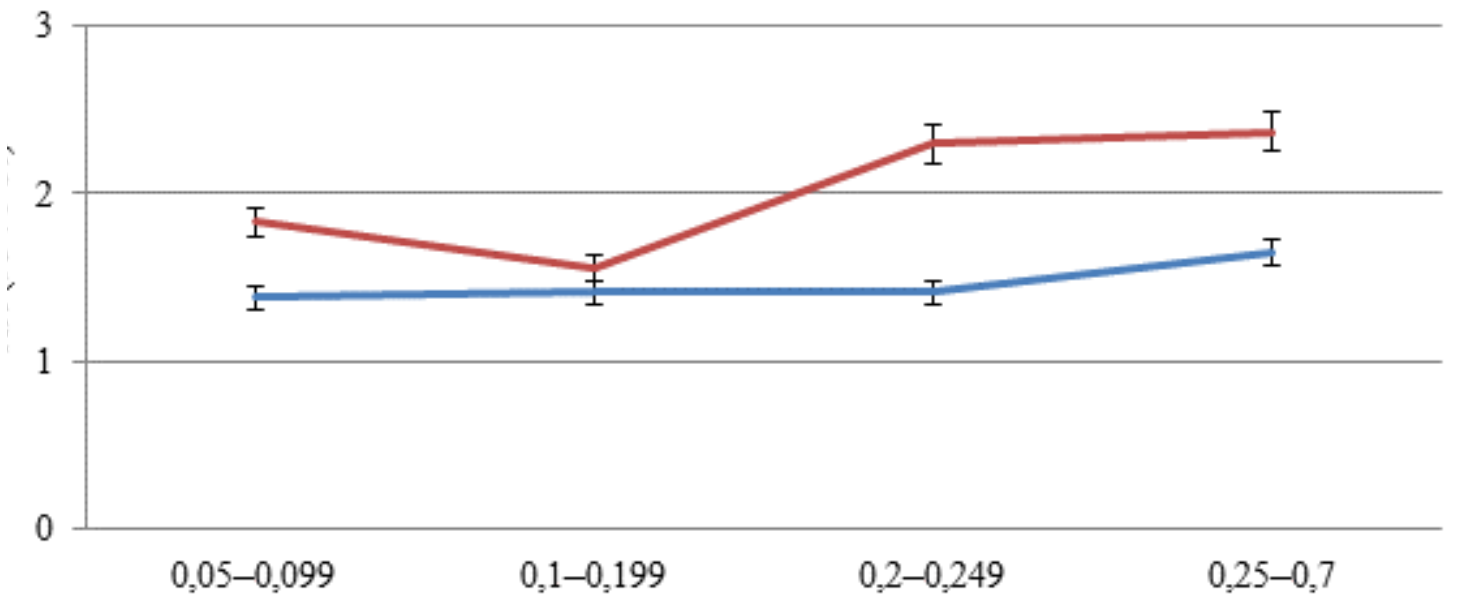

- 18-39 years old at the exposure Dose, Gy

(ICD-9: 290-319, ICD-10: F00-F99

Conclusions: There are consequent epidemiological evidences on radiation cerebral effect as organic brain damage in liquidators, as well as acute and chronic cerebrovascular pathology. 